\title{
Emergent Lorentz Symmetry with Vanishing Velocity in a Critical Two-Subband Quantum Wire
}

\author{
M. Sitte ${ }^{1}$, A. Rosch ${ }^{1}$, J. S. Meyer ${ }^{2}$, K. A. Matveev ${ }^{3}$, and M. Garst ${ }^{1}$ \\ ${ }^{1}$ Institut für Theoretische Physik, Universität zu Köln, Zülpicher Straße. 77, 50937 Köln, Germany \\ ${ }^{2}$ Department of Physics, The Ohio State University, Columbus, Ohio 43210, USA \\ ${ }^{3}$ Materials Science Division, Argonne National Laboratory, Argonne, Illinois 60439, USA
}

(Dated: May 20, 2018)

\begin{abstract}
We consider a quantum wire with two subbands of spin-polarized electrons in the presence of strong interactions. We focus on the quantum phase transition when the second subband starts to get filled as a function of gate voltage. Performing a one-loop renormalization group analysis of the effective Hamiltonian, we identify the critical fixed-point theory as a conformal field theory having an enhanced SU(2) symmetry and central charge 3/2. While the fixed point is Lorentz invariant, the effective "speed of light" nevertheless vanishes at low energies due to marginally irrelevant operators leading to a diverging critical specific heat coefficient.

PACS numbers: 71.10.Pm, 64.70.Tg, 75.40.Cx
\end{abstract}

As a function of an external gate voltage, the conductance of quantum wires increases in steps of height $G_{0}=2 e^{2} / h$ when more and more subbands are subsequently filled. Each conductance plateau transition defines a quantum critical point where the ground state changes its character. Close to each critical point one expects universal scaling and a rich behavior as a function of gate voltage and temperature. These quantum phase transitions and especially their transport signatures are, however, only partially understood [1, 2, 3, 4].

A quantum phase transition is characterized by the critical degrees of freedom, their dynamics, and the underlying symmetries at the critical point [5]. In onedimensional systems, many quantum critical points are Lorentz invariant, and conformal field theory (CFT) provides a valuable scheme to classify them. In such a case, the powerful machinery available for CFTs allows the calculation of the critical behavior and the systematic investigation of the leading corrections to scaling. In particular, a characteristic of CFTs is a linear specific heat, $C=c(\pi / 3 v) T$, inversely proportional to the velocity $v$ of excitations, that identifies the central charge $c$ measuring the effective number of degrees of freedom [ $[$, , 7].

In this Letter we study a model of spinless fermions close to the quantum critical point where a second subband is about to get filled as a function of gate voltage. Three different phases [2, 3] are relevant to describe this system. First, for a small chemical potential, only the first subband is occupied, and the electron system forms an ordinary Luttinger liquid. Second, for a large chemical potential, both subbands are filled, and one obtains two Luttinger liquids. There is, however, also a third phase which can be visualized as a (fluctuating) zigzag Wigner crystal, where the relative motion of charges in the two subbands is locked and only one gapless mode survives.

Upon increasing the chemical potential, the onedimensional phase undergoes a transition to the fluctuating zigzag Wigner crystal phase [2, 3]. The nature of this transition depends on the interaction strength. For weak interactions, it has been argued [1, 2] that thermodynamic properties at the critical point, where the first phase becomes unstable, are not qualitatively changed by the interactions (see below). One obtains a Lifshitz transition describing the filling of a noninteracting subband of fermions with a quadratic dispersion. More precisely, the interactions turn out to be dangerously irrelevant. While they do not affect the properties at criticality, they nevertheless induce a zigzag Wigner crystal for small but finite filling of the second subband. When the interactions are sufficiently strong, however, the universality class of the transition changes. The identification of the critical properties at such strong interactions is the aim of this Letter.

We show that the corresponding transition has a rather unusual structure. On the one hand, we find that the critical fixed point is given by a well-known CFT with central charge $c=3 / 2$, describing three Majorana fermions with equal velocity. As in many other systems the symmetries at the critical point are enhanced as neither Lorentz invariance nor the $\mathrm{SU}(2)$ symmetry of the $c=3 / 2 \mathrm{CFT}$ are properties of the original model. On the other hand, it turns out that, at criticality, the effective velocity - the "speed of light" of the CFT - vanishes in the low-energy limit. This peculiar behavior arises from marginally irrelevant corrections to the CFT that spoil Lorentz invariance. It is thus not a property of the fixed point itself, but a consequence of the slow flow towards this fixed point. We discuss the conditions under which a vanishing of the critical velocity occurs generically. A direct consequence of this behavior is a divergent critical specific heat coefficient, $\gamma=C / T$, for $T \rightarrow 0$.

A known example of a similar situation occurs in a model for two-dimensional electrons in graphene interacting via a long-range Coulomb potential [8, 9]. The Fermi points in graphene give rise to an emergent Lorentz invariance that is, however, broken by the static Coulomb 
interaction. Because of the vanishing density of states at the Fermi points, the interaction remains unscreened and thus long-range. Although the effective interaction is found to be marginally irrelevant, Lorentz invariance remains broken in the low-energy limit. In contrast to our case, the effective velocity is logarithmically enhanced in the presence of Coulomb interaction, resulting in a suppression of the specific heat.

To be specific, we study the model Hamiltonian $\mathcal{H}=$ $\mathcal{H}_{1}+\mathcal{H}_{2}+\mathcal{H}_{12}$, where [2]

$$
\begin{gathered}
\mathcal{H}_{1}=\frac{v}{2 \pi} \int d x\left[K\left(\partial_{x} \theta\right)^{2}+\frac{1}{K}\left(\partial_{x} \phi\right)^{2}\right] \\
\mathcal{H}_{2}=\int d x \psi^{\dagger}\left(-\frac{\partial_{x}^{2}}{2 m}-\mu+\mu_{\mathrm{cr}}\right) \psi \\
\mathcal{H}_{12}=\int d x\left[-\frac{g_{x}}{\pi} \partial_{x} \phi \psi^{\dagger} \psi+\frac{u}{2}\left(e^{i 2 \theta} \psi \partial_{x} \psi+\text { H.c. }\right)\right] .
\end{gathered}
$$

The first subband [see Fig. 1(a)] has a finite filling and can be treated as a Luttinger liquid [Eq. (10]. Its excitations are plasmons, propagating waves of electron density, $\delta n=-\frac{1}{\pi} \partial_{x} \phi$, represented by the bosonic field $\phi$, and $\theta$ is its conjugate with $\left[\frac{1}{\pi} \partial_{x} \phi(x), \theta\left(x^{\prime}\right)\right]=-i \delta\left(x-x^{\prime}\right)$. The second subband, on the other hand, contains spinless non-relativistic fermions [Eq. (2)] that are close to the quantum phase transition occurring at $r \equiv \mu_{\mathrm{cr}}-\mu=0$ when $\mu$ is increased (e.g., by tuning a gate voltage). Near this transition, the interaction among fermions in the second subband is negligible due to the Pauli principle. The Galilean invariance of $\mathcal{H}_{2}$ is manifest and implies a critical dynamical exponent $z=2$ for the fermions in the absence of intersubband interactions. However, there are two important types of such interactions. The first term in Eq. (3) is the density-density interaction, $g_{x}$, and the second term, $u$, arises from pair-tunneling processes of electrons as depicted in Fig. 1(a). We assume $u \geq 0$. Interestingly, the nature of the transition at $r=0$ was found to depend on the interaction strength [2].

First, consider the Hamiltonian $\mathcal{H}$ without pair tunneling, $u=0$. At criticality, $r=0$, there is a van Hove
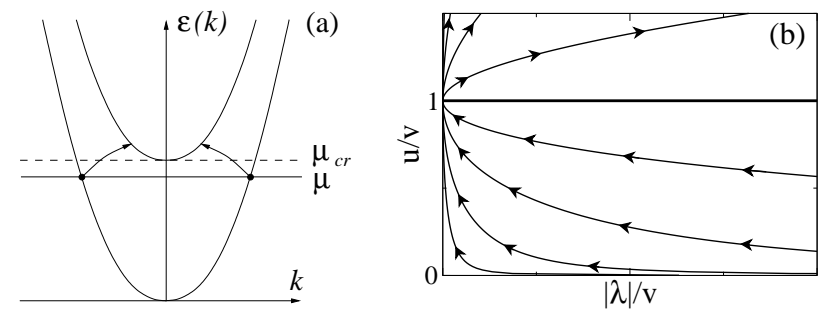

FIG. 1: (a) Pair-tunneling process between the two subbands. The chemical potential $\mu$ is close to the critical value $\mu_{\mathrm{cr}}(r \equiv$ $\left.\mu_{\text {cr }}-\mu\right)$. (b) One-loop RG flow diagram of the effective model $\mathcal{L}_{\text {eff }}$ determined by the invariant $\mathcal{I}=\lambda^{2} K v u /(u-v)^{4}$ [see Eqs. (8) and (9)]. $\lambda$ is the interaction, $u$ is the pair tunneling and $v$ is the plasmon velocity. There is a line of fixed points at $u=v$ parametrized by the dynamical exponent (7). singularity in the tunneling density of states (DOS) that results in a strong hybridization between the modes of the two subbands in the presence of a finite interaction, $g_{x}$. At their band bottom, the fermions are very slow, and the fast plasmons can adiabatically adjust themselves to the fermions in the second subband. Explicit calculations [1] show that the coupling of the resulting dressed fermions to the Luttinger liquid is irrelevant at the critical point. Therefore the transition remains a usual onedimensional Lifshitz transition. For $\mu<\mu_{\mathrm{cr}}$ the chemical potential is below the band edge, implying a charge gap $\Delta=\mu_{\mathrm{cr}}-\mu=r$. In the limit of a weak interaction $g_{x}$, this scenario remains valid even in the presence of a finite pair tunneling $u$. However, it was observed in Ref. [2], that, for $r<0$, the pair tunneling $u$ leads to a ground state with a zigzag charge density wave associated with a gap, $\Delta \sim(-r)^{\alpha}$, where the exponent is large, $\alpha \gg 1$, in the weak coupling limit. As a consequence, the actual conductance plateau transition does not coincide with the Lifshitz transition at $r=0$, but is rather shifted to a negative value of $r<0$, i.e., a finite filling of the second subband [3].

The universality class of the transition at $r=0$ changes qualitatively when the interaction $g_{x}$ increases. To understand the nature of this transition, we apply a unitary transformation, $U=e^{i \int \theta \psi^{\dagger} \psi d x}$, to the Hamiltonian such that effectively $\psi \rightarrow U^{\dagger} \psi U=\psi e^{-i \theta}$ and $\partial_{x} \phi \rightarrow \partial_{x} \phi+\pi \psi^{\dagger} \psi$. Switching to a Euclidean Lagrangian description, the effective model then can be written as $\mathcal{L}_{\text {eff }}=\mathcal{L}_{\text {LL }}+\mathcal{L}_{\text {Ising }}+\mathcal{L}_{\text {int }}$, where

$$
\begin{gathered}
\mathcal{L}_{\mathrm{LL}}=\frac{1}{2 \pi v K}\left[\left(\partial_{\tau} \phi\right)^{2}+v^{2}\left(\partial_{x} \phi\right)^{2}\right], \\
\mathcal{L}_{\text {Ising }}=\psi^{\dagger} \partial_{\tau} \psi+\frac{u}{2}\left(\psi \partial_{x} \psi+\text { H.c. }\right)+r \psi^{\dagger} \psi, \\
\mathcal{L}_{\text {int }}=-\lambda \frac{1}{\pi} \partial_{x} \phi \psi^{\dagger} \psi,
\end{gathered}
$$

$\tau$ is the imaginary time, and $\lambda \equiv g_{x}-\pi v / K$.

For $\lambda=0$, the plasmonic and fermionic sectors decouple, but the fermionic part $\mathcal{L}_{\text {Ising }}$ can now be identified with an Ising model that is critical for $r=0$. The term $\psi^{\dagger} \partial_{x}^{2} \psi$ in Eq. (2) is negligible close to criticality as it is subleading compared with the spatial gradient term in $\mathcal{L}_{\text {Ising. }}$. As a consequence, an effective Lorentz invariance for the critical fermionic excitations emerges, leading to a dynamical exponent $z=1$. Note, however, that there are two different velocities: the fermions propagate with velocity $u$ and the plasmons with velocity $v$. Moreover, the effective model now possesses the self-duality symmetry of the Ising model which guarantees that, for $\lambda=0$, there is a gap $\Delta=|r|$ in the fermionic sector on either side of the transition.

In the present work, we analyze the effective model $\mathcal{L}_{\text {eff }}$ perturbatively in the residual interaction $\lambda[10$, which explicitly breaks the Lorentz invariance of both the plasmonic and the fermionic excitations. We show 
that this Lorentz-invariance breaking leaves traces in the critical behavior although the interaction $\lambda$ is found to be (marginally) irrelevant in the renormalization group (RG) sense.

Perturbation theory in $\lambda$ is accompanied by logarithmic IR singularities that can be absorbed into a renormalization group flow of the parameters of the model. Introducing a momentum cutoff $\Lambda$, we perform a Wilsonian renormalization group step by integrating out perturbatively modes within a momentum shell $(\Lambda / b, \Lambda)$ with $b>1$, and we calculate the plasmon and fermion selfenergy and the vertex correction to one-loop order (see Fig. (2). After rescaling momenta $k \rightarrow k / b$ and frequencies $\omega \rightarrow \omega / b^{z}$, where $z$ is a so-far-unspecified dynamical exponent, the momentum cutoff of the perturbatively corrected theory is restored to $\Lambda$. Moreover, introducing wave function renormalizations, $\phi \rightarrow \sqrt{Z_{B}} \phi$ and $\psi \rightarrow \sqrt{Z_{F}} \psi$, we can choose the RG conditions such that the bosonic velocity $v$, the Luttinger parameter $K$, and the unit prefactor of $\psi^{\dagger} \partial_{\tau} \psi$ in (5) are RG invariants. This can be achieved by introducing an anomalous dynamical exponent

$$
z=1+\frac{\lambda^{2} K}{4 \pi^{2} u v}
$$

A dynamical exponent $z \neq 1$ is a manifestation of the broken Lorentz invariance. Note that within this RG scheme the velocities $u$ and $v$ are not the physical velocities. In particular, the plasmon velocity $v_{\text {phys }}$ flows under RG (see below).

The fermionic velocity $u$, the interaction $\lambda$, and the effective chemical potential $r$ obey the one-loop RG equations:

$$
\begin{gathered}
\frac{\partial u}{\partial \ln b}=-\frac{\lambda^{2} K}{\pi^{2}}\left[\frac{1}{(u+v)^{2}}-\frac{1}{4 u v}\right] u \\
\frac{\partial \lambda}{\partial \ln b}=-\frac{\lambda^{3} K}{2 \pi^{2}}\left[\frac{1}{u(v+u)}+\frac{1}{(u+v)^{2}}-\frac{3}{4 u v}\right] \\
\frac{\partial r}{\partial \ln b}=r-\frac{r \lambda^{2} K}{2 \pi^{2}}\left[\frac{1}{u(v+u)}+\frac{1}{(u+v)^{2}}-\frac{1}{2 u v}\right] .
\end{gathered}
$$

These coupled nonlinear differential equations can be solved analytically by realizing that the quantity $\mathcal{I}=$ $\lambda^{2} \mathrm{Kvu} /(u-v)^{4}$ is an invariant of the RG flow. $\mathcal{I}$ determines directly the flow diagram shown in Fig. 1 (b). If

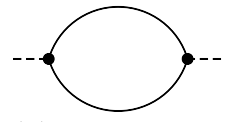

(a)

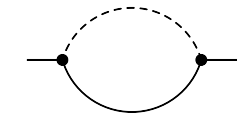

(b)

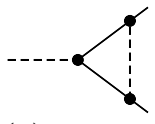

(c)
FIG. 2: One-loop perturbative corrections. The dashed and solid lines represent the plasmon and fermion propagator, respectively. The dot is the interaction vertex $\lambda$ given in Eq. (6). (a) Plasmon self-energy, (b) fermion self-energy, and (c) vertex-correction. the fermionic velocity is larger than the bosonic velocity, $u>v$, the RG flow is towards strong coupling, $\lambda \rightarrow \infty$, which may indicate either a first order transition or a different strong coupling fixed point. If the fermionic and bosonic velocities coincide, $u=v$, the one-loop beta functions (8) and (9) vanish, and one obtains a line of fixed points parametrized by the dynamical exponent $z$ [Eq. (7)]. This line of fixed points is, however, likely to be an artifact of the one-loop RG. Fortunately, in a two-subband quantum wire we expect the pair-tunneling $u$ to be much smaller than the plasmon velocity of the first subband $v[10]$. In this regime, the RG flow is towards weak coupling, $\lambda \rightarrow 0$, and is fully controlled by our one-loop analysis [11].

If the flow is towards weak coupling, the fermionic velocity $u(b)$ approaches the bosonic velocity $v$ in the limit $b \rightarrow \infty$ [see Fig. 1(b)]. As a consequence, the weakcoupling fixed point is characterized by a single velocity. Diagonalizing the fermionic sector and refermionizing the bosonic mode of the effective model $\mathcal{L}_{\text {eff }}$ for $\lambda=0$ and $u=v$, the fixed-point Hamiltonian can be expressed in terms of three left- and right-moving Majorana fields, $L_{a}$ and $R_{a}$, respectively:

$$
\mathcal{H}_{\mathrm{FP}}=\frac{i}{2} \sum_{a=1}^{3} v\left[L_{a} \partial_{x} L_{a}-R_{a} \partial_{x} R_{a}\right] .
$$

At the critical point one has an enhanced SU(2) symmetry describing a rotation in the space of the three real Majorana fields [12]. Furthermore, the fixed point is Lorentz invariant. Nevertheless, we show below that the residual breaking of Lorentz invariance due to the interaction $\lambda$ leads to important corrections to the fixed-point Hamiltonian (11), although $\lambda$ is formally irrelevant.

The flow towards the fixed point can be determined by considering the limit of a small velocity difference: $w \equiv 1-u / v \ll 1$. By expanding the RG equations (8) and (9) in lowest order in $w$, one finds

$$
\frac{\partial w}{\partial \ln b}=-\frac{K}{16 \pi^{2} v^{2}} \lambda^{2} w^{2}, \quad \frac{\partial \lambda}{\partial \ln b}=-\frac{K}{8 \pi^{2} v^{2}} \lambda^{3} w .
$$

In this regime, $u$ approaches $v$ very slowly,

$$
u(b) \approx v\left(1-\frac{\mathcal{A}}{(\ln b)^{1 / 5}}\right),
$$

where the prefactor $\mathcal{A}=\left(16 \pi^{2} / 5 \mathcal{I}\right)^{1 / 5}$ has been obtained from the exact solution of (8) and (9).

If the pair tunneling is very small, $u \ll v$, there is an extended regime where the initial flow differs qualitatively from the asymptotic behavior (12):

$$
u(b) \approx \sqrt{u^{2}+v^{2} \frac{\mathcal{I}}{2 \pi^{2}} \ln b} .
$$

An estimate of the crossover scale $b^{*}$ between the two regimes yields $\ln b^{*} \sim 1 / \mathcal{I}$. This scale is very large in 


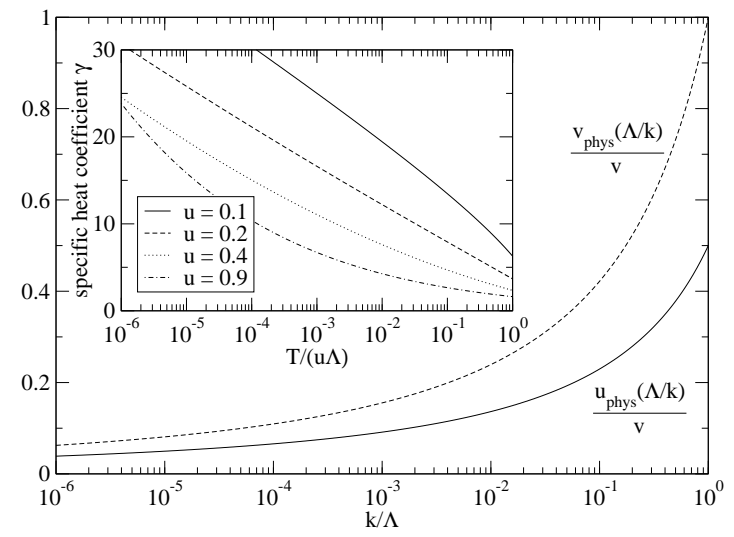

FIG. 3: Momentum dependence of the physical velocities of the Luttinger liquid, $v_{\text {phys }}(\Lambda / k)$, and the excitations of the second subband, $u_{\text {phys }}(\Lambda / k)$, for $K=1,|\lambda|=v=1$ and $u=$ 0.5 at the quantum critical point. For $k \rightarrow 0$ both velocities vanish while $u_{\text {phys }}(\Lambda / k) / v_{\text {phys }}(\Lambda / k) \rightarrow 1$. Inset: specific heat coefficient for various values of $u$.

the limit $u \ll v$ such that the asymptotic behavior is attained only at extremely low energies.

When interpreting the results given above, it is essential to realize that they have been obtained in an RG scheme where time and space have been rescaled in an asymmetric way as $z \neq 1$. As velocity is length over time, this asymmetric scaling modifies the physical velocities by an extra factor

$$
\Omega(b)=\exp \left[\int_{0}^{\ln b}\left(1-z\left(b^{\prime}\right)\right) d \ln b^{\prime}\right]
$$

with $v_{\text {phys }}=\Omega v, u_{\text {phys }}=\Omega u$. For low energies, i.e., $\ln b \rightarrow \infty, z(b)$ approaches unity because the fixed point is Lorentz invariant:

$$
z(b) \approx 1+\frac{2}{5}\left(\frac{10 \mathcal{I}}{\pi^{2}}\right)^{1 / 5} \frac{1}{(\ln b)^{4 / 5}} .
$$

However, the flow towards $z=1$ is so slow that the integral $\int\left[z\left(b^{\prime}\right)-1\right] d \ln b^{\prime}$ in (15) diverges and, thus, $\Omega(b \rightarrow \infty)=0$. This implies that at the fixed point, $\ln b=\infty$, all physical velocities vanish. In Fig. 3 the momentum dependence of the two physical velocities $u_{\text {phys }}$ and $v_{\text {phys }}$ is shown. As described by Eq. (13) the two velocities approach each other slowly, and both vanish for $k \rightarrow 0$ due to the $z$ renormalization of Eq. (15).

The vanishing of the velocities naturally leads to a divergence of the specific heat coefficient $\gamma$. At criticality $(r=0), \gamma$ scales as the inverse of the physical velocities, $\gamma(b)=\frac{\pi}{6 \Omega(b)}\left(\frac{2}{v}+\frac{1}{u(b)}\right)$. The RG flow is stopped when $T(b) / u(b)$ reaches the cutoff, where temperature obeys $\partial T / \partial \ln b=z T$. From Eqs. (15) and (16), one then obtains asymptotically

$$
\gamma \sim \exp \left[2\left(\frac{10 \mathcal{I}}{\pi^{2}} \ln \frac{v \Lambda}{T}\right)^{1 / 5}\right] .
$$

The inset of Fig. 3 shows the $T$ dependence of $\gamma$.

Away from criticality, $r \neq 0$, the fermionic excitations are gapped. The gap $\Delta$ can be obtained by integrating the RG equations (8)-(10) up to $b=b_{\Delta}$ with $r\left(b_{\Delta}\right)=$ $u\left(b_{\Delta}\right) \Lambda$. In physical units, this corresponds to the energy scale $\Delta=u\left(b_{\Delta}\right) \Omega\left(b_{\Delta}\right) \Lambda / b_{\Delta}$, where we have again taken into account the rescaling of the time axis. Compared to the Ising relation, $\Delta=|r|$, the resulting $T=0$ gap,

$$
\Delta \sim|r| \exp \left[-3\left(\frac{10 \mathcal{I}}{\pi^{2}} \ln \frac{v \Lambda}{|r|}\right)^{1 / 5}\right]
$$

is suppressed due to the residual interaction $\lambda$. The suppression is, however, slower than power law. The gap $\Delta$ should also be observable in the tunneling DOS.

In this Letter we have shown that the quantum phase transition from a Luttinger liquid to a zigzag Wigner crystal has a fixed point given by a Lorentz-invariant theory of three noninteracting Majorana fermions. However, the effective velocities of the modes vanish for low energies. This behavior will occur quite generally for systems where marginally irrelevant terms break the Lorentz invariance. More precisely, whenever the correction to the dynamical critical exponent vanishes with $1 / \ln b$ or slower, the integral in (15) diverges such that - depending on the sign of $(z-1)$ - the effective velocity will either diverge or vanish for low energies.

We thank N. Andrei, L. Balents, T. Senthil, and M. Vojta for useful discussions. This work was supported by the DFG through SFB 608 and by the U. S. Department of Energy, Office of Science, under Contract No. DEAC02-06CH11357 and No. DE-FG02-07ER46424.

[1] L. Balents, Phys. Rev. B 61, 4429 (2000).

[2] J. S. Meyer et al., Phys. Rev. Lett. 98, 126404 (2007).

[3] J. S. Meyer and K. A. Matveev, J. Phys. Condens. Matter 21, 023203 (2009).

[4] M. Garst et al., Phys. Rev. B 77, 035128 (2008).

[5] H. v. Löhneysen et al., Rev. Mod. Phys. 79, 1015 (2007).

[6] H. W. J. Blöte et al., Phys. Rev. Lett. 56, 742 (1986).

[7] I. Affleck, Phys. Rev. Lett. 56, 746 (1986).

[8] A. A. Abrikosov and S. D. Beneslavskii, Zh. Eksp. Teor. Fiz. 59, 1280 (1970) [Sov. Phys. JETP 32, 699 (1971)].

[9] J. González et al., Phys. Rev. B 59, R2474 (1999).

[10] For quantum wires with Coulomb interaction screened by a gate at distance $d$, the parameters $g_{x}, v$, and $K$ are related [2], yielding $\lambda \simeq-\pi v K=-\pi v_{F 1}$. The perturbative $\mathrm{RG}$ in $\lambda$ is controlled by the small parameter $\lambda^{2} K /(v u) \sim v_{F 1}^{2} /\left[e^{4} \log \left(k_{F 1} d\right)\right]$. Here $v_{F 1}$ and $k_{F 1}$ is the Fermi velocity and momentum of the filled band, respectively, and $\log \left(k_{F 1} d\right) \gg 1$. Note that the same estimates give $(u / v)^{2} \sim e^{2} /\left[v_{F 1} \log \left(k_{F 1} d\right)\right] \ll 1$ for the ratio of velocities.

[11] The one-loop RG is controlled at least as long as $v-u>$ $\sqrt{K}|\lambda|$, which follows from the worst-case assumption that the two-loop corrections to the RG equations (12) are of order $\mathcal{O}\left((v-u)^{0}\right)$.

[12] A. M. Tsvelik, Phys. Rev. B 42, 10499 (1990). 